\title{
Frequency and Prognosis of Epidermal Growth Factor Receptor Variant III Mutations in Glioblastoma Multiforme among Indian Patients: A Single-Institution Study
}

Wesley Mannirathil Jose ${ }^{1} \quad$ Vinayak Munirathnam ${ }^{1} \quad$ V. Narendranath ${ }^{2} \quad$ Arun Philip $^{1}$

Pavithran Keechilat ${ }^{1}$

${ }^{1}$ Department of Medical Oncology and Hematology, Cancer Institute, Amrita Institute of Medical Sciences, Amrita Vishwa Vidyapeetham, Kochi, Kerala, India

2Department of Molecular Medicine, Amrita Institute of Medical Sciences, Amrita Vishwa Vidyapeetham, Kochi, Kerala, India
Address for correspondence Dr. Wesley Mannirathil Jose, MD DNB, Department of Medical Oncology and Hematology, Cancer Institute, Amrita Institute of Medical Sciences, Amrita Vishwa Vidyapeetham, Kochi, Kerala, India (e-mail: wesleymjose@aims.amrita.edu)

\section{South Asian J Cancer 2020;9:126-129.}

Abstract

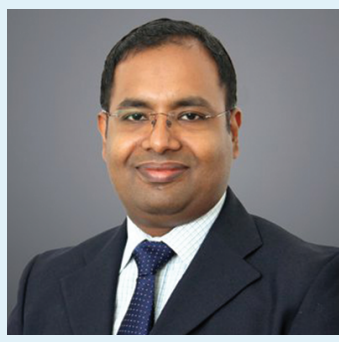

Dr. Wesley Mannirathil Jose, MD DNB

Keywords

- epidermal growth factor receptor

- glioblastoma multiforme

- mutation

- variant III
Background Glioblastoma multiforme (GBM) is a disease with poor outcome. Alterations or mutations in epidermal growth factor receptors (EGFRs) are found in GBM and may be targeted to improve outcomes.

Aims We analyzed the frequency of EGFR variant III (vIII) mutations in patients with GBM and their outcomes after standard treatment.

Materials and Methods This is a retrospective study conducted in a single tertiary cancer center in south India. Forty patients with GBM who had their entire treatment done at this center were identified, and their primary tumor tissue blocks were retrieved. Genomic DNA was extracted, and molecular analysis was performed and analyzed. The results of mutational analysis were correlated with treatment outcome of the patients.

Statistical Analysis Survival outcome was analyzed using the Kaplan-Meier method. The log-rank test was used to assess the association between the groups and various parameters.

Results Our study showed a similar incidence of EGFR vIll alterations as published in world literature, but we did not find any difference in overall survival (OS) and progression-free survival (PFS) in patients with EGFR vIll mutation compared with nonmutant cohort.

Conclusions Contrary to the existing literature which indicated EGFR vIll alterations to be a negative prognostic indicator, our study did not find it to be an independent predictor of prognosis among Indian GBM patients treated with present standard of care.
How to cite this article: Jose WM, Munirathnam V, Narendranath V, Philip A, Keechilat P. Frequency and Prognosis of Epidermal Growth Factor Receptor Variant III Mutations in Glioblastoma Multiforme among Indian Patients: A Single-Institution Study. South Asian J Cancer 2020;9(3):126-129.

DOI https://doi.org/10.1055/s-0041-1723078 ISSN 2278-330X.
(C) 2020. MedIntel Services Pvt Ltd.

This is an open access article published by Thieme under the terms of the Creative Commons Attribution-NonDerivative-NonCommercial-License, permitting copying and reproduction so long as the original work is given appropriate credit. Contents may not be used for commercial purposes, or adapted, remixed, transformed or built upon. (https://creativecommons.org/licenses/by-nc-nd/4.0/)

Thieme Medical and Scientific Publishers Pvt. Ltd., A-12, 2nd Floor, Sector 2, Noida-201301 UP, India 


\section{Introduction}

Glioblastoma multiforme (GBM) and its variants (giant cell glioblastoma and gliosarcoma) are the most malignant forms of primary neuroepithelial tumors of the central nervous system (CNS) and fall under the purview of WHO Grade IV in the neuropathological classification schemes. ${ }^{1}$ The American Brain Tumor Association data for 2008-2012 reported that in the United States, GBM accounts for 12 to $15 \%$ of all intracranial tumors and 50 to $60 \%$ of astrocytic tumors. ${ }^{2}$ In India, a prospective study of 656 patients with CNS neoplasms from Tata Memorial Hospital reported 38.7\% ( $n=254)$ gliomas, and among these, 59.5\% (151/254) were high-grade gliomas. ${ }^{3}$ Since India does not have a wide-based population cancer registry, the hospital cancer registry data may provide only a skewed understanding of incidence and mortality. GBM can affect patients at any age but has a peak incidence between the ages of 45 and 75 years. Our own institutional unpublished data show the median age to be 51.5 years (16-75 years).

GBM has the poorest overall survival (OS), with only $0.05-4.7 \%$ of patients surviving 5 years past their diagnosis. ${ }^{4}$ Chromosomal alterations are a common reason for gliomagenesis. Molecular, cytogenetic, and array-based assays of comparative genomic hybridization and RNA expression have opened doors to the understanding of genetic alterations which are likely to be causative of gliomas. ${ }^{5}$

It has been established that the transformed phenotype of brain tumor cells is highly complex and results from the dysfunction of a variety of interrelated regulatory pathways. The transformation process involves the amplification or overexpression of oncogenes and loss or lack of expression of tumor suppressor genes. Abnormalities in TP53 (mutation) and epidermal growth factor receptor (EGFR) (amplification) have shown correlations with the occurrence of GBM. ${ }^{6}$ In the past two decades, there has been significant literature on the prognostic relevance of EGFR, in particular its receptor variant III (EGFR vIII). Glioblastomas often express EGFR vIII, which is a constitutively active genomic deletion variant of EGFR and is characterized by deletions of exons 2-7 of the EGFR gene. EGFR vIII characterized by the truncated extracellular domain has a ligand-independent constitutive activity and confers increased proliferation and invasiveness to glioma cells. ${ }^{7}$

We analyzed the frequency of EGFR VIII mutations among our patients afflicted with GBM to assess the presence of molecular alterations and its impact on the disease in our patient population.

\section{Materials and Methods}

This is a single-center, nonrandomized, retrospective study in patients diagnosed with GBM. Only those GBM patients who received their entire treatment at this tertiary care hospital between January 2014 and August 2015 and had documented follow-up records until the time of study initiation were included. This was done to ensure the uniformity of treatment received by the patients. Patients who were lost to follow-up or those with clinical outcomes not available were excluded from the study. A total of 40 patient tumor samples were identified and included in the study. The protocol was reviewed and approved by the institutional review board.

The formalin-fixed paraffin-embedded (FFPE) tumor blocks were retrieved from the department of pathology archives and were tested for EGFR vIII mutation. Tissue sections of $5-\mu \mathrm{m}$ thickness were obtained from FFPE blocks and stained with methyl green. The tumor-rich areas were microdissected using a $21 \mathrm{G}$ needle, and the samples were subjected to proteinase $\mathrm{K}$ digestion in a rotating incubator at $56^{\circ} \mathrm{C}$ for 3 days. Genomic DNA was extracted using the DNeasy Tissue Kit (Qiagen, Hilden, Germany) and kept at $4^{\circ} \mathrm{C}$ before use.

SALSA MLPA probemix was used to determine the deletions of EGFR which results in EGFR vIII. Samples were tested as per the manufacturer's protocol. The deletions and duplications detected by multiplex ligation-dependent probe amplification (MLPA) were not confirmed by other methods due to financial constraints. MLPA analysis was done on isolated tumor DNA; hence, the changes detected in our study are essentially at the DNA level. The results of mutational analysis were correlated with patient demographics and treatment outcome of the patients.

Descriptive statistics were used to describe patient characteristics as frequencies. The actual values relating to the patient characteristics are mentioned in mean or median values. Statistical analysis was performed using the IBM SPSS version 20 software. Survival outcome analysis was done using the Kaplan-Meier method. Association between the groups and various parameters (age, extent of surgical excision, size of the tumor, etc.) was looked at using the logrank test.

\section{Results}

This retrospective study included 40 patients. All patients received conventional treatment with maximal safe resection, followed by chemoradiation and adjuvant temozolomide. The radiation dose was $6000 \mathrm{cGy}$ in 30 fractions over a period of 6 weeks. The concurrent temozolomide was dosed at $75 \mathrm{mg} / \mathrm{m}^{2}$ and the adjuvant was administered at 150 to $200 \mathrm{mg} / \mathrm{m}^{2} 1$ to 5 days every 4 weeks for six cycles.

Twenty-four (60\%) patients were male. EGFR vIII mutation was detected in 23 (57.5\%) tumor samples. The sex distribution among the mutant group was identical; however, it was male predominant ( $n=12 ; 70.6 \%)$ in the nonmutant population. The mean age in the EGFR vIII-positive patients was 47.13 years, which was approximately 5 years lower than 52 years in the negative group. The most common site of tumor in EGFR vIII-mutant patients was temporal region. EGFR vIII-mutant tumors were relatively larger than their negative counterparts. In spite of the larger size, the total plus near-total surgical resection rates were identical in both the groups. 
The median progression-free survival (PFS) time among the whole cohort was 10.53 months. The median PFS time in the EGFR vIII-positive group was 11.10 months, and in the EGFR vIII-negative group, it was 8.83 months ( $p=$ 0.520) (-Fig. 1). The median OS time among the whole cohort was 16.7 months. The median OS time in the EGFR

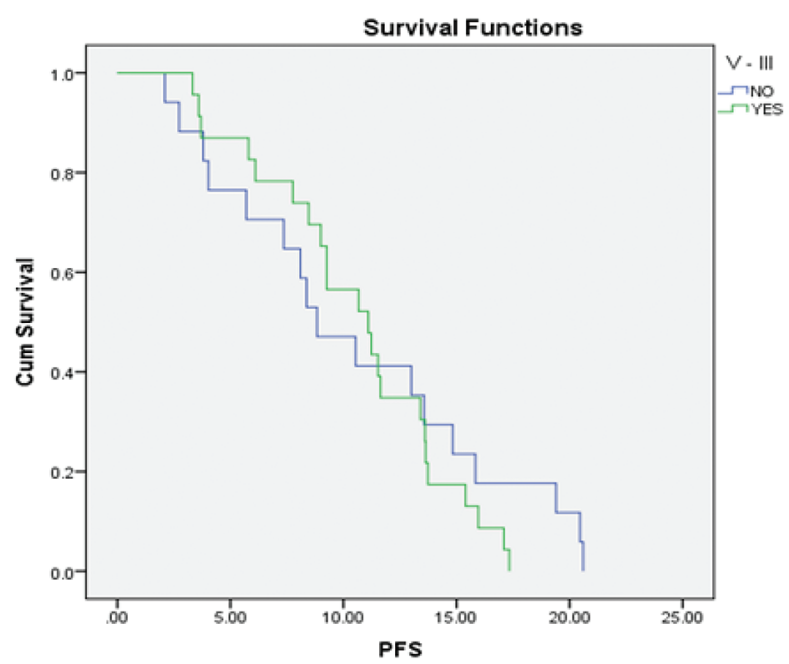

Fig. 1 Kaplan-Meier graph for progression-free survival (PFS) time in epidermal growth factor (EGFR) receptor variant III (vIII).

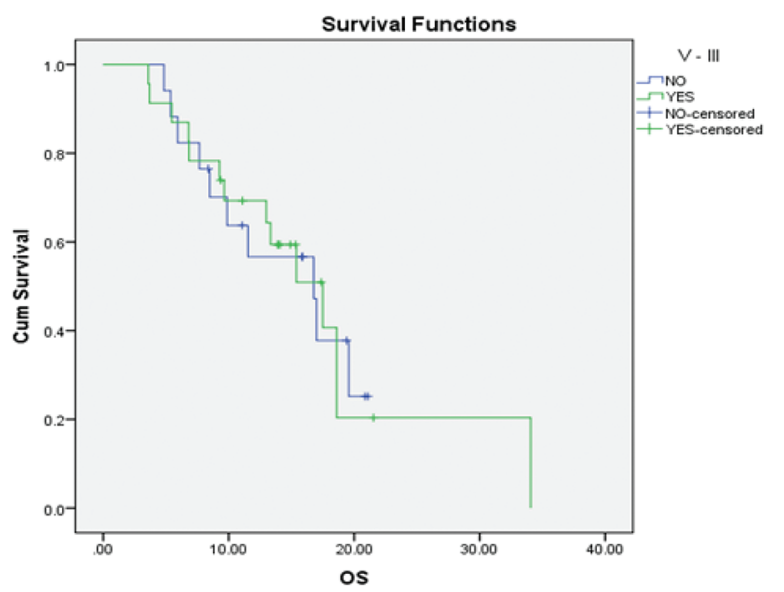

Fig. 2 Kaplan-Meier graph for overall survival (OS) time in epidermal growth factor receptor (EGFR) variant III (vIII).
vIII-positive group was 17.5 months, and in the negative group, it was 16.76 months ( $p=0.953$ ) ( $\sim$ Fig. 2 ). There was no statistically significant difference between the survivals of these two groups.

\section{Discussion}

GBM is a disease with a very short median survival, elucidating that both prognostic and predictive biomarkers play a very important role. Approximately 54\% of GBMs with EGFR gene amplification also express EGFR vIII. ${ }^{8}$ The EGFR vIII-positive cells are located in cellular areas of the tumor but not in the invading zone. ${ }^{9}$ This could suggest that EGFR vIII has a central tumor-initiating and perpetuating role. The upregulated EGFRs participate in key processes of tumor cell invasion and tumor-related angiogenesis. Glioma cell invasion and increased angiogenesis share mechanisms of the degradation of the extracellular matrix (ECM) through the upregulation of ECM-degrading proteases as well as the activation of aberrant signaling pathways. $^{10}$

The frequency of GBM with EGFR vIII mutation at 57.5\% in our cohort was in line with the available literature. ${ }^{7,11}$ Analysis of data from the groups carrying EGFR vIII mutation as a variable showed no statistical difference in the OS and the PFS. We have represented for comparison some of the available studies in literature in - Table $1 .{ }^{11-13}$ The fact that $57.5 \%$ of patients expressed EGFR vIII, and they had a median age of 47 years, was interesting.

Even though the presence of EGFR vIII did not translate into a poor prognosticator factor in our cohort, it still would find therapeutic applications. A recent publication from our institution has demonstrated the significance of humanized anti-EGFR vIII single-chain fragment variable as a potential immunotherapeutic agent as well as a targeting agent for specific delivery of drugs to EGFR vIII-expressing cancer cells. ${ }^{14}$ Work on orthotopic mice models has suggested that tumor-selective, bitargeted anti-EGFR/EGFR vIII CAR T-cells may be a promising modality for the treatment of patients with EGFR/EGFR vIII-overexpressing GBM. ${ }^{15}$

The limitation of our study is its small sample size. Due to cost constraints, we have not been able to verify the positive samples using other real-time polymerase chain reaction-based assays or next-generation sequencing to confirm the alterations identified in our study.

Table 1 Few comparable studies for EGFR alterations

\begin{tabular}{|l|l|l|l|}
\hline Study author/years & Number of cases & EGFR study & Result \\
\hline Shinojima et al ${ }^{11}$ & 87 & EGFR vlll alterations & $\begin{array}{l}\text { Significant unfavorable predictor on OS for amplification. } \\
\text { EGFR vlll showed a trend toward shorter OS }\end{array}$ \\
\hline Heimberger et al ${ }^{12}$ & 196 & EGFR vlll alterations & No prognostic significance on OS with EGFR vlll \\
\hline${\text { Umesh et al }{ }^{13}}^{13}$ & 54 & Alterations & Significant negative prognostic factor on OS \\
\hline Present study & 40 & Alterations of EGFR vlll & No impact of EGFR vill mutation on the OS and PFS \\
\hline
\end{tabular}

Abbreviations: EGFR vIII, epidermal growth factor receptor variant III; OS, overall survival; PFS, progression-free survival. 


\section{Conclusions}

Based on our study, in the Indian context among patients with GBM, EGFR mutations are present and carry prognostic significance. However, EGFR vIII does not translate into poor prognosis. However, in a cost-constrained setting, we believe that EGFR vIII identification may identify patients who could benefit from newer therapeutic measures.

\section{Financial Support and Sponsorship}

Nil.

Conflicts of Interest

None declared.

\section{References}

1 Louis DN, Perry A, Reifenberger G, et al. The 2016 world health organization classification of tumors of the central nervous system: A summary. Acta Neuropathol 2016;131(6):803-820

2 Ostrom QT, Gittleman H, de Blank PM, et al. American brain tumor association adolescent and young adult primary brain and central nervous system tumors diagnosed in the united states in 2008-2012. Neuro-oncol 2016;18(Suppl 1) :i1-i50

3 Jalali R, Datta D. Prospective analysis of incidence of central nervous tumors presenting in a tertiary cancer hospital from India. J Neurooncol 2008;87(1):111-114

4 Ostrom QT, Bauchet L, Davis FG, et al. The epidemiology of glioma in adults: a "state of the science" review. Neuro-oncol 2014;16(7):896-913

5 Ohgaki H, Dessen P, Jourde B, et al. Genetic pathways to glioblastoma: a population-based study. Cancer Res 2004;64(19): 6892-6899
6 von Deimling A, Louis DN, Wiestler OD. Molecular pathways in the formation of gliomas. Glia 1995;15(3):328-338

7 Aldape KD, Ballman K, Furth A, et al. Immunohistochemical detection of EGFRvIII in high malignancy grade astrocytomas and evaluation of prognostic significance. J Neuropathol Exp Neurol 2004;63(7):700-707

8 Liu L, Bäcklund LM, Nilsson BR, et al. Clinical significance of EGFR amplification and the aberrant EGFRvIII transcript in conventionally treated astrocytic gliomas. J Mol Med (Berl) 2005;83(11):917-926

9 Nozawa T, Okada M, Natsumeda M, et al. EGFRvIII is expressed in cellular areas of tumor in a subset of glioblastoma. Neurol Med Chir (Tokyo) 2019;59(3):89-97

10 Keller S, Schmidt MHH. EGFR and EGFRvIII promote angiogenesis and cell invasion in glioblastoma: Combination therapies for an effective treatment. Int J Mol Sci 2017;18(6):e1295

11 Shinojima N, Tada K, Shiraishi S, et al. Prognostic value of epidermal growth factor receptor in patients with glioblastoma multiforme. Cancer Res 2003;63(20):6962-6970

12 Heimberger AB, Hlatky R, Suki D, et al. Prognostic effect of epidermal growth factor receptor and EGFRvIII in glioblastoma multiforme patients. Clin Cancer Res 2005;11(4):1462-1466

13 Umesh S, Tandon A, Santosh V, et al. Clinical and immunohistochemical prognostic factors in adult glioblastoma patients. Clin Neuropathol 2009;28(5):362-372

14 Xavier S, Gopi Mohan C, Nair S, Menon KN, Vijayachandran LS. Generation of humanized single-chain fragment variable immunotherapeutic against EGFR variant III using baculovirus expression system and in vitro validation. Int J Biol Macromol 2019;124:17-24

15 Jiang H, Gao H, Kong J, et al. Selective targeting of glioblastoma with EGFRvIII/EGFR bitargeted chimeric antigen receptor T cell. Cancer Immunol Res 2018;6(11):1314-1326 